\title{
Ecological optimization design of silage equipment key components based on innovation method theory
}

\author{
Jianqiang Wang, Bing Lva , Jie Yan, Yingjun Li, Zequn Li and Mengjia Chen \\ School of Mechanical Engineering, University of Jinan, 250022 Jinan Shandong, China
}

\begin{abstract}
Ecological environmental issues are constantly threatening the survival and development of human society and has been concerned by all walks of life. The seven ecological efficiency factors proposed by the WBCSD are the basis for improving the ecological performance. Through the mapping relationship between ecological efficiency factors and 39 engineering parameters, the ecological issues will be transferred to engineering problems. It is guided by the corresponding design concepts. Furtherly the TRIZ problem solving tools will be applied. Thereby, the optimized solution will be obtained. It can not only effectively reduce the environmental impact of products, but also can shorten the development cycle of green products while satisfying functional requirements. The development of China's agricultural machinery is extensive. This method is used to implement ecological optimization design of the bundling device mounting frame for silage equipment, which has important guiding significance for the development of China's agricultural machinery.
\end{abstract}

\section{Introduction}

Since the industrial development of our country to date, great changes have taken place in human society: science and technology have advanced by leaps and bounds, economic strength has been continuously enhanced, and material conditions have greatly improved. At the same time, as the rapid development of society, people have to face more and more serious resource and environmental problems [1]. The ratio of industrial output capacity and resource-environmental cost are low in China, especially in the field of agricultural machinery. At present, the development of large-scale agricultural machinery, high cost, energy-consuming supplies, but the low utilization rate, does not meet China's crop planting patterns and farmers' income levels.

The construction of ecological civilization is related to everyone. The traditional development model of development-pollution-government has failed in China. In the 1960s, when the book "The Silent Spring" was published, people began to pay attention to the ecological value of design. Z.F. Liu et al. [2] combined CBR with TRIZ to summarize the green product design model; F. Fei et al. [3] proposed 8 environmental parameters, and then transformed them into TRIZ problems through the relationship with engineering parameters; L. Zhang et al [4] obtained the product green design process by making up for the lack of TRIZ and patent analysis. In the conceptual design phase of the product, we take into account the ecological impact combining innovation method theory with ecological design. It can shorten the development cycle of green product.
With the adjustment of agricultural structure and the continuous development of animal husbandry, the acreage of silage crops has been expanding year by year. The demand for silage equipment is also increasing. However, the development of silage equipment is still in the traditional stage of development. This paper introduces ecological design into the field of agricultural machinery. It applies further an ecological design model based on the innovation method theory to analyze silage equipment so that silage equipment can improve its ecological performance while fulfilling its functions and meeting customer needs.

\section{Ecological design based innovation method theory}

\subsection{Theoretical basis}

The innovation method is an auxiliary tool for improving people's innovative skills based on a large number of case studies and practices. It includes TRIZ, SIT and QFD [5]. Particularly, TRIZ is a powerful methodology for solving invention problems. Eco-design theory is mainly used to guide the direction of product development. The innovation method theory and the ecological design theory have become more mature. The method of ecological design based on innovation method theory has emerged. 


\subsubsection{TRIZ theory}

G.S. Altshuller from the former Soviet Union and a group of researchers led by him analyzed and studied 2.5 million patents from countries in the world [6]. In turn, they proposed TRIZ theory. TRIZ has three basic solutions [7]: conflict resolution principles, effects, and 76 standard solutions. Conflict includes technical conflict and physical conflict. Technical conflicts apply invention principles to solve problems by conflict matrix, and the physical conflict apply invention principles to solve problems by the separation principle. The effect is to solve problems with the scientific principles of physics, chemistry, and mathematics. The application of the 76 standard solutions is to establish the material-field problem model and then find the corresponding solution model.

\subsubsection{Ecological design}

Ecological design refers to not only considering the performance, price, market and appearance of the product in the conceptual design stage of the product, but also considering the ecological performance of the product from the entire life cycle. It maximizes the use of product resources and minimizes ecological impact [8].

As part of the product's overall design, eco-design is not a specific method or tool, but rather a way of thinking and analysing. It is a necessary precautionary measure. Eco-design sometimes provides users with an exciting need. It is not as easy to discover as basic needs and expected needs. Once it is realized, it will greatly increase the market competitiveness of products.

\subsection{Ecological design method based on TRIZ}

In order to further improve the level of sustainable development and strengthen the awareness of ecological environmental protection, the World Business Council for Sustainable Development (WBCSD) has proposed 7 ecological efficiency factors [9]: reducing the material intensity of products and services; reducing the energy intensity of products and services; reducing the spread of toxic substances; improving the recyclability of raw materials; promoting the sustainable use of renewable resources; improving the durability of products; increasing the service intensity of goods. In order to facilitate the use of operations, they are represented by A, $\mathrm{B}, \mathrm{C}, \mathrm{D}, \mathrm{E}, \mathrm{F}, \mathrm{G}$ respectively. The introduction of ecological efficiency factors also provides a basis for the ecological design of products.

Chen et al. [10] summarized the mapping relationship between 7 ecological efficiency factors of WBCSD and 39 engineering parameters of TRIZ. In addition, a association table of ecological efficiency elements and engineering parameters was established, as shown in Table 1. Through this table, we will quickly turn ecological issues into TIRZ engineering problems. So it provides method and theoretical guidance for problem solving. And products can quickly and effectively improve the ecological efficiency factors.

Table 1. Ecological efficiency factors and engineering parameters association table.

\begin{tabular}{|c|c|}
\hline $\begin{array}{c}\text { Ecological } \\
\text { efficiency } \\
\text { factors }\end{array}$ & TRIZ engineering parameters \\
\hline A & $1,2,3,4,5,6,7,8,12,14,23,26,32,39$ \\
\hline B & $1,3,5,7,17,18,19,20,21,22,32,39$ \\
\hline C & $13,23,26,28,30,31$ \\
\hline D & $9,10,11,28,29,32,36$ \\
\hline E & $14,30,31,34$ \\
\hline F & $9,24,25,27,33,34,35,37,38,39$ \\
\hline G &
\end{tabular}

Because an ecological efficiency factor does not correspond to an engineering parameter. In order to facilitate the selection of engineering parameters, the design concept corresponding to the ecological efficiency factors is summarized for the ecological design of product structure in the mechanical field, as shown in Table 2. This is based on the relationship between ecological design concepts, environmental demand characteristics and green attributes [11]. It guides the selection of TRIZ engineering parameters. Ecological design analyzes one of the seven factors that affect ecological efficiency. But it does not mean that ecological efficiency factors can be isolated. Conversely, the impact on other factors must be fully considered in designing.

Table 2. Ecological efficiency factors and design concepts.

\begin{tabular}{|c|c|}
\hline $\begin{array}{c}\text { Ecological } \\
\text { efficiency } \\
\text { factors }\end{array}$ & Design concepts \\
\hline A & Minimal design, Lightweight design \\
\hline B & Energy-saving design \\
\hline C & Minimal pollution design \\
\hline D & $\begin{array}{c}\text { Modular design, Design for recyclability, } \\
\text { Design for disassembly }\end{array}$ \\
\hline E & $\begin{array}{r}\text { Resource best use design, Resource } \\
\text { regeneration design }\end{array}$ \\
\hline F & Long life design, Balanced life design \\
\hline G & Reduced design, Modular design \\
\hline
\end{tabular}

Ecological efficiency factors can guide products to reduce negative impacts. TRIZ theory can provide a more direct and easier-to-operate theoretical basis. Integrating eco-efficiency factors and TRIZ, the ecological design model based on innovative method theory is obtained. Figure 1 shows. It not only guides the design direction but also provides method theory. 


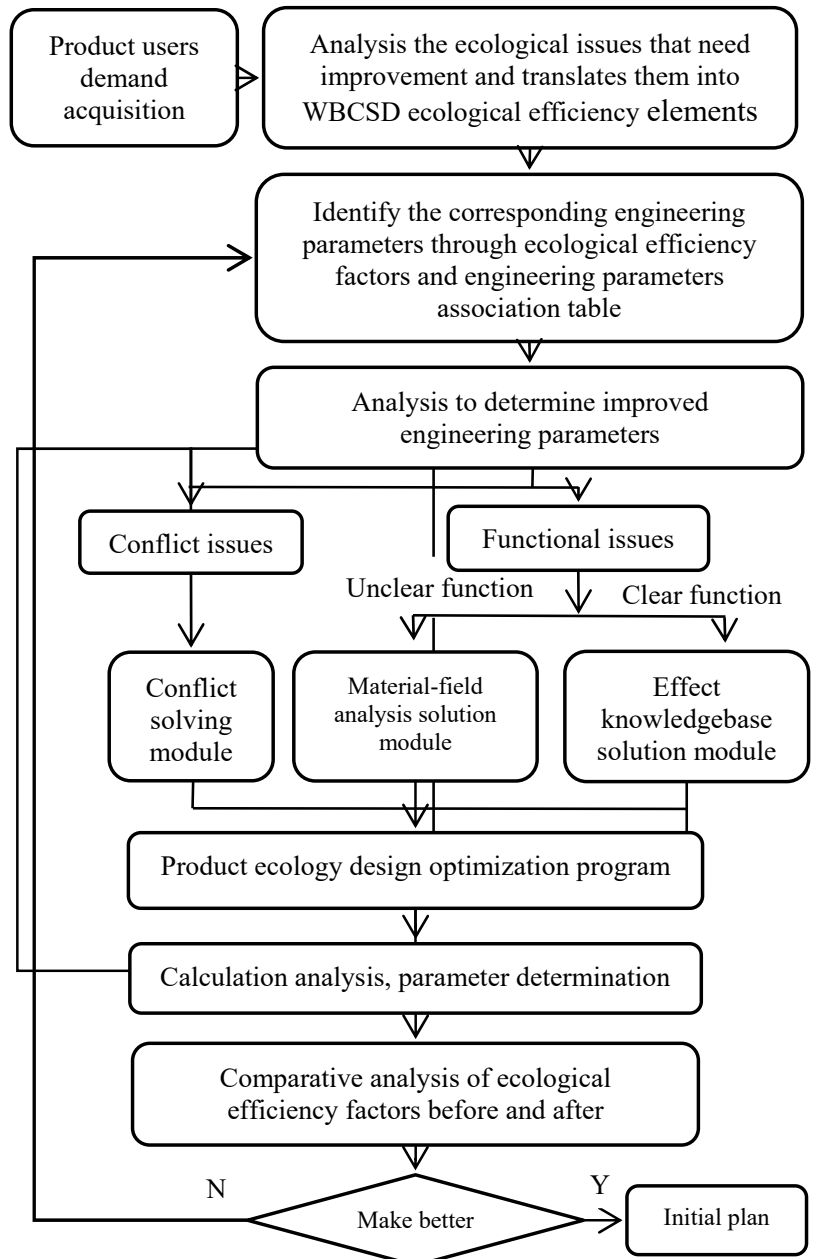

Figure 1. Ecological design model based on innovation method theory.

The ecological design model mainly includes six parts: demand analysis, ecological analysis, problem solving, preliminary program, parameter determination and program evaluation. The solution to the problem is to turn the actual problem into a model problem. And then selecting the corresponding solution principle, problem solving model or scientific effect applies to the specific problem.

Eco-design based on the innovation method theory can not only improve the product's environmental friendliness, but also improve the market competitiveness of products and shorten the development cycle of green products while satisfying the needs of customers. Therefore, the application of ecological design method based on TRIZ theory is of great significance for the optimization design of products.

\section{Ecological optimization design of silage equipment}

In 2017, the total sown area of grain was 112,220 thousand hectares in China, which was 815 thousand hectares less than last year. The increase in feed grain poses a threat to Chinese food security. It also restricts the development of animal husbandry in China [12]. Silage expands the source of feed and can solve the above problems. Therefore, the research on silage equipment is of great significance.

\subsection{Overall structure of silage equipment}

China is a big country in agriculture and animal husbandry. However, it is insufficient that agricultural machinery is suitable for hilly areas. The existing knapsack silage equipment has a relatively single function. But power resources are wasted and the cost of material transportation is high in each step. There is a difference in process time, which affects the silage effect. In order to overcome the shortcoming of the current silage machinery. We developed modular knapsack silage equipment that integrates cutting, smashing, baling, and wrapping. It mainly includes a header device, a throwing device, a bundling device, a wrapping device, a lifting device, a telescopic device, and a power device.

This equipment is small and medium-sized and harvests two rows. The width of the header device is 1.4 $\mathrm{m}$. The working speed is $5 \mathrm{~km} / \mathrm{h}$. The main working parameters are determined as follows:

(1) straw quality per hectare

We calculate corn yields at $6000 \mathrm{~kg} / \mathrm{hm}^{2}$. the ratio of corn straw material quality and corn kernel quality is $1.2: 1$. So we calculated the straw quality to be $7200 \mathrm{~kg}$ per hectare. $80 \%$ of the corn stalks are recycled and $20 \%$ of the crop stalks are returned to the field. The straw quality collected was $5760 \mathrm{~kg}$ per hectare. Therefore, the quality of the grain and straw collected was:

$$
m=6000+5760=11760 \mathrm{~kg} .
$$

The moisture content of silage straw is generally $40 \%$ to $60 \%$. If we take $50 \%$, straw quality per hectare was:

$$
m_{g}=m / 0.5=23520 \mathrm{~kg} \text {. }
$$

(2) Working time per hectare

The row spacing of corn is $0.6 \mathrm{~m}$ and the harvest width is $1.2 \mathrm{~m}$. The working time per hectare was:

$$
t_{g}=\frac{1000}{\frac{5000}{3600} \times 1.2}=1.667 \mathrm{~h} .
$$

(3) Stem harvesting productivity

The theoretical calculation of stem harvesting productivity [13] was:

(1)

$$
\begin{array}{lll}
Q_{j} & = & 0.1 q_{j} W v_{j}
\end{array}
$$

Where: $W=$ width of work, $\mathrm{m} ; \mathrm{q}_{j}=$ stem yield, $\mathrm{kg} / \mathrm{hm}^{2}$; and $v_{j}=$ machine travel speed, $\mathrm{km} / \mathrm{h}$.

Calculated:

$$
Q_{j}=0.1 \times 1.2 \times 23520 \times 5=3.92 \mathrm{~kg} / \mathrm{s} .
$$




\subsection{Installation structure design}

In order to make full use of resources and improve the utilization of equipment, mounting frame for bundling device play a key role in the modular design. The ecological optimization design of the mounting frame can improve the use performance and ecological performance of the entire equipment.

\subsubsection{Demand analysis}

The existing mounting frames are tailored to the tractor and can only be adapted to the specific model tractor. When the power unit is replaced, it cannot be used. The bundling device is difficult to install on the mounting frame and requires engineering equipment.

\subsubsection{Ecological analysis}

The adaptability of the mounting frame is poor. It needs to be re-customized for different tractors. Thus this wastes material. And it cannot achieve self-handling so much that it needs to use engineering equipment. Thus this wastes resources. We model the actual problem. It corresponds to the eco-efficiency factor: increasing the service intensity of goods $(\mathrm{G})$. According to Table 1, TRIZ engineering parameters corresponding to ecological efficiency factor $\mathrm{G}$ are $9,24,25,27,33,34$, $35,37,38$, and 39. Based on reduced design concept, the improvement project parameters 33 are selected after the analysis. Operability: the operations to be completed require fewer operators and steps, and the use of tools are as simple as possible.

\subsubsection{Problem solving}

After the ecological analysis, it is known that the engineering parameters that need to be improved are operability. In other words, the mounting frame can achieve as many functions as possible with as little manpower and time as possible. Whether it is the installation of the bundling device or the installation of the mounting frame, their displacements are realized, which is a function problem. And the function is clear. Therefore, the effect is used to solve the problem. According to the function code table, its corresponding function code is F6 (controlling the displacement of the object). The recommended scientific effects include magnetic force, electronic force, pressure and inertia force. Pressure is applied to the movement of the mounting frame, which make the mounting frame scalable. Inertial force is used for the movement of the bundling device, which save human effort.

\subsubsection{Preliminary plan}

In order to facilitate the installation and adjustment of the mounting frame, we use square tube nesting and install the hydraulic cylinder inside the square tube. So the installation frame is adjustable and controllable in the three-dimensional space. In order to achieve the self- loading and unloading of the bundling device, a slide rail is provided on the support plate. Slides are used with the slider on the bundling device. Of course, the lifting device for mounting the wrapping device is also fully utilized to achieve self-loading and unloading. Figure 2 shows a retractable mounting frame.

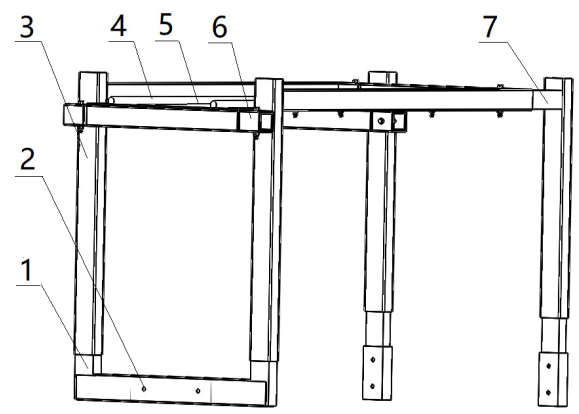

1. corner tube 2 . mounting hole 3 . hydraulic cylinder 4 . slide rail 5. support plate 6 . connection tube 7 . built-in tube

Figure 2. the retractable mounting frame

\subsubsection{Determination of main parameters}

The equipment is small or medium sized. Mounting frame is mainly suitable for tractors of 50/60/70/80 horsepower different brands. It is mounted on the hood of the tractor. The design length can be adjusted in the range of $1400-1800 \mathrm{~mm}$; the width can be adjusted in the range of $600-1000 \mathrm{~mm}$; the height can be adjusted in the range of $800-1300 \mathrm{~mm}$.

\section{Summary}

(1) This paper applies the ecological design method based on innovation method theory to optimize the key components of silage equipment. The ecological performance analysis and the working performance analysis of silage equipment will be completed in the follow-up work.

(2) The model integrated innovation method theory and eco-design method was applied to the field of agricultural machinery, which is of great significance to the healthy and effective development of agricultural machinery.

\section{Acknowledgment}

Fund Project: 2017 Shandong Province Agricultural Machinery R\&D and Innovation Plan Funded Project (2017YF004-10)

\section{References}

1. Z.F. Liu, Green Design Method Technology and Its Application (Nat. Def. Ind. Press, Beijing, 2008)

2. Z.F. Liu, Y. Gao, D. Hu, J.D. Zhang, China. Mech. Eng, 23, 9: 1105-1116 (2012)

3. F. Fei, L.W. Zhong, Precis. Manufa. Automat, 2: 4756 (2008) 
4. L. Zhang, W.W. Zhang, S.X. Jiang, Mech. Des. Res, 32, 5: 1-3 (2016)

5. C.Y. He, Y. Li, W.Q. Li, J. Jiang, J. Eng. Des, 18, 3: 153-158 (2011)

6. R.H. Tan, Q.H. Zhang, Ind. Eng. Manage, 1: 34-36 (2003)

7. M. Zhao, W.C. Zhang, G.S. Wang, TRIZ Advanced and Practical (Mech. Ind. Press, Beijing, 2016)

8. J.N. Liu, G. Jiang, W.J. Lu, X.D. Zhang, J. Mech. Eng, 52, 5: 12-20 (2016)

9. L.D. Desimone, F. Popoff, Eco-efficiency: The Business Link to Sustainable Development (MA. Inst. Techn, Cambridge, 1997)

10. J.L. Chen, C.C. Liu. J. Sustain. Prod. Des, 1, 4: 263$272(2003)$

11. G.C. Ren, Y.L. Tang. Mech. Des. Manu, 12: 61-63 (2016)

12. Z.Q. Wang, S.M. Teng, M. Zhao. Chinese Cow, 2: 43-44 (2007)

13. China Academy of Agricultural Mechanization, Agricultural Machinery Design Manual (Mach. Ind. Press, 1988) 